\title{
Rapid Presentation Rate Negatively Impacts the Contiguity Effect in Free Recall
}

\author{
Claudio Toro-Serey (ctoro@bu.edu) and Ian M. Bright (imbright@bu.edu) \\ Department of Psychological and Brain Sciences, 64 Cummington Mall \\ Boston, MA 02215 USA \\ Brad P. Wyble (bpw10@psu.edu) \\ Department of Psychology, 140 Moore Building \\ University Park, PA 16801 USA \\ Marc W. Howard (marc777@bu.edu) \\ Department of Psychological and Brain Sciences, 64 Cummington Mall \\ Boston, MA 02215 USA
}

\begin{abstract}
It is well-known that in free recall participants tend to recall words presented close together in time in sequence, reflecting a form of temporal binding in memory. This contiguity effect is robust, having been observed across many different experimental manipulations. In order to explore a potential boundary on the contiguity effect, participants performed a free recall task in which items were presented at rates ranging from $2 \mathrm{~Hz}$ to $8 \mathrm{~Hz}$. Participants were still able to recall items even at the fastest presentation rate, though accuracy decreased. Importantly, the contiguity effect flattened as presentation rates increased. These findings illuminate possible constraints on the temporal encoding of episodic memories.
\end{abstract}

Keywords: Free recall, lag-CRP, contiguity effect

\section{Introduction}

Cognitive neuroscientists have hypothesized that the successful retrieval of an episodic memory is accompanied by a "jump back in time," a recovery of the previous memory's spatiotemporal context (Tulving, 1983). In free recall studies, this recovery manifests as the contiguity effect, wherein following the successful recall of an item, the next item to be recalled is more likely to be a close temporal neighbor than a more distant one (Kahana, 1996). This distance is measured as lag, a directed distance between items in a study list. For example, in the list "absence, hollow, pupil, river, darling", the lag from absence to river is +3 , while the lag from darling to pupil is -2 . In free recall studies the contiguity effect is typically asymmetric, such that forward transitions are more likely to take place than backward transitions of the same distance. This effect is robust, appearing across a variety of methodological manipulations (Kahana, 2012; Healey \& Kahana, 2014). For instance, the contiguity effect is observed with more or less the same properties for lists of different modalities (Kahana, 1996), when rehearsal is discouraged (Howard \& Kahana, 1999), and when words are widely separated in time (Howard, Youker, \& Venkatadass, 2008; Unsworth, 2008). Healey and Kahana (2014) noted that the contiguity effect was observed for every individual participant in a free recall study of 126 subjects. Thus far, dramatic effects on the contiguity effect in free recall have primarily been observed comparing patient populations; older adults and memory disordered individuals show impaired contigu- ity effects (Kahana, Howard, Zaromb, \& Wingfield, 2002; Palombo, Di Lascio, Howard, \& Verfaellie, 2019).

Beyond the contiguity effect, free recall contains many other well-explored patterns of behavior. Individuals exhibit a strong recency effect during immediate free recall tests (Glanzer \& Cunitz, 1966). In addition, participants exhibit a primacy effect such that items at the beginning of a studied list are more likely to be recalled (B. B. Murdock, 1962). Both primacy and recency effects are observed in the initiation of free recall, and are both also robustly observed in the probability of first recall, a measure of the serial position curve considering only the first recall (Hogan, 1975; Laming, 1999). The relative strength of primacy and recency is not constant however (B. B. Murdock, 1962). For example, Davelaar, Goshen-Gottstein, Ashkenazi, Haarmann, and Usher (2005) found that presentation rates affect the relative strength of primacy and recency, with primacy becoming more prevalent as the presentation rate is increased.

The ubiquity of the contiguity effect in free recall presents something of a challenge for models of memory encodingif nothing affects the contiguity effect, it makes it more difficult to understand how it comes about. Conversely, if we knew boundary conditions on the contiguity effect it would perhaps shed light on the processes supporting the binding of experiences presented close together in time. In this study we explore the effects of increasing presentation rates on the contiguity effect. If the contiguity effect is disrupted at a particular rate, that suggests the time scale over which the encoding processes necessary for temporal binding take place.

Considerations from the ERP literature and rapid serial visual presentation (RSVP) literature inform the time scale over which contiguity might be disrupted. A to-be-remembered stimulus typically evokes a P300 waveform approximately $500 \mathrm{~ms}$ in duration that is thought to represent the updating of memory representations, even when the stimulus duration itself is on the order of 2 seconds (Donchin, 1981). At presentation rates approaching $10 \mathrm{~Hz}$, there is evidence that individual list items are no longer processed as discrete items, and instead are merged into a single extended cognitive event. For example, individual items in $10 \mathrm{~Hz}$ lists receive very low hit rates in an immediate recognition test even 
when the stimuli are never-before-seen natural images (Potter \& Levy, 1969). This poor performance is in stark contrast to the excellent recognition memory for long series of images at slower rates of presentation (Standing, 1973; Brady, Konkle, Alvarez, \& Oliva, 2008). However despite this lack of memorability, it is also clear that each item in a $10 \mathrm{~Hz}$ stream is processed to some degree, since it is possible to detect specific target items with high probability (Potter, 1976). If the processing of individual items in a list undergoes a qualitative change as the presentation rate is increased to the point at which the representations blend together, then the CRP, primacy effect, and recency effect may be altered. For example, the CRP effect may depend on the ability to place individual items into a discrete temporal representation, and thus it may disappear with faster presentation rates. The probability of first recall could also be altered, since a long-running stream of rapidly presented items imposes a sequential cost on subsequent items due to encoding interference from previous items (Wyble, Bowman, \& Nieuwenstein, 2009).

\section{Methods}

\section{Participants}

Three hundred and thirty undergraduates from Syracuse University participated in this study. Participants were excluded if they failed to recall a correct word in at least one trial $(n=15)$, and if they did not perform all three conditions $(n=7)$. Data from 308 participants were used in subsequent analyses.

\section{Procedure}

Participants took part in 18 trials. Each trial consisted of 20 words from the Toronto Noun Pool (Friendly, Franklin, Hoffman, \& Rubin, 1982). Words were visually displayed at three presentations rates: $2 \mathrm{~Hz}, 4 \mathrm{~Hz}$, and $8 \mathrm{~Hz}$. Participants completed six trials in each condition. Trial order was randomized. Before the start of a trial, participants viewed a bar that discretely rotated at the same rate that words would be presented to help orient them to the upcoming trial (e.g., before a $2 \mathrm{~Hz}$ trial the bar would move twice every second). Following the presentation of the list, participants were prompted to verbally recall as many words as possible from the list. Responses were recorded and later parsed using a semi-automatic speech parsing algorithm.

\section{Analysis}

We first examined whether presentation rate affected the average number of valid recalls in a trial. This was done with a repeated measures ANOVA. Post-hoc paired permutations (5000 iterations) and Cohen's D effect sizes on mean recalls were then performed to determine significant differences. Serial position curves (SPC) were computed to show the overall probability of a word being recalled based on its position in the list for each participant. We examined whether the recency and primacy effects changed as a function of presentation rate. We performed a paired permutation test in order

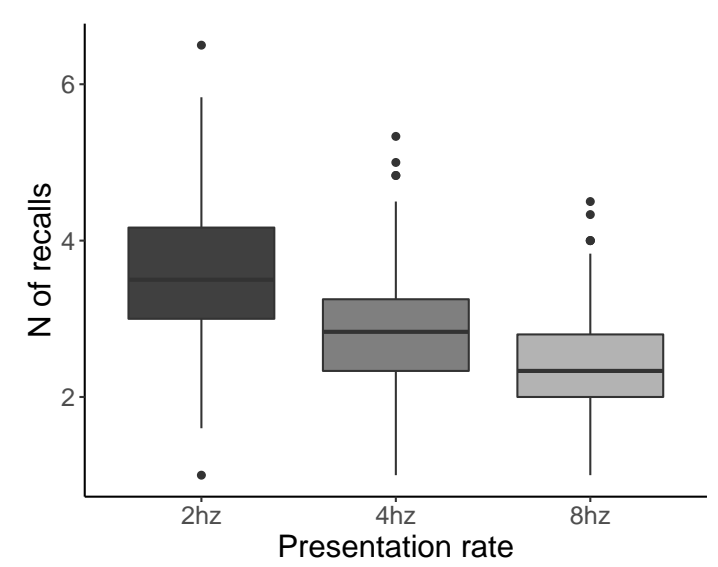

Figure 1: A boxplot of median number of words recalled per trial across participants and presentation rates, with interquartile range, $95 \%$ confidence intervals and outliers. Participants recalled fewer words as presentation rate increased.

to predict the difference in the probability of recall for the first and last items in the list (i.e., probability for position 20 minus probability for position 1).

The probability of first recall (PFR) was calculated by dividing the number of times each serial position was recalled first by the total number of first recalls. We then averaged these probabilities across participants per condition. Finally, we calculated the conditional response probability (CRP) for each lag by dividing the number of correct recall transitions at a given lag by the total number of possible correct transitions at that lag. In order to control for serial position effects, which differed across conditions, we restricted the lag-CRP analysis to transitions within the middle of the list where probability of recall was approximately equal across presentation rates. In order to test for differences in the CRP at each lag across conditions, we performed a number of mixed-effects logistic regressions. We estimated the CRP as a function of the interaction between the following fixed-effects predictors: absolute lag, its direction (backwards or forwards from the previously recalled item), and presentation rate. We report $\mathrm{Z}$ - and T-scored coefficients for all mixed-effects models.

\section{Results}

To anticipate the results, memory performance was reduced at faster presentation rates. We replicated previous findings with respect to changes in the serial position curve at fast presentation rates. Critically, the contiguity effect, even measured at serial positions that avoided contributions from primacy and recency, was severely disrupted at fast presentation rates.

\section{As Presentation Rate Increases, Fewer Words are Recalled}

As shown in Figure 1, the total number of words recalled decreased as presentation rates increased $(2 \mathrm{~Hz}$ : mean $=3.54$, $\mathrm{SD}=0.85 ; 4 \mathrm{~Hz}$ : mean $=2.86, \mathrm{SD}=0.662 ; 8 \mathrm{~Hz}$ : mean $=2.41, \mathrm{SD}=0.62$; ANOVA: $F(2,614)=309.2, p<0.001)$. 


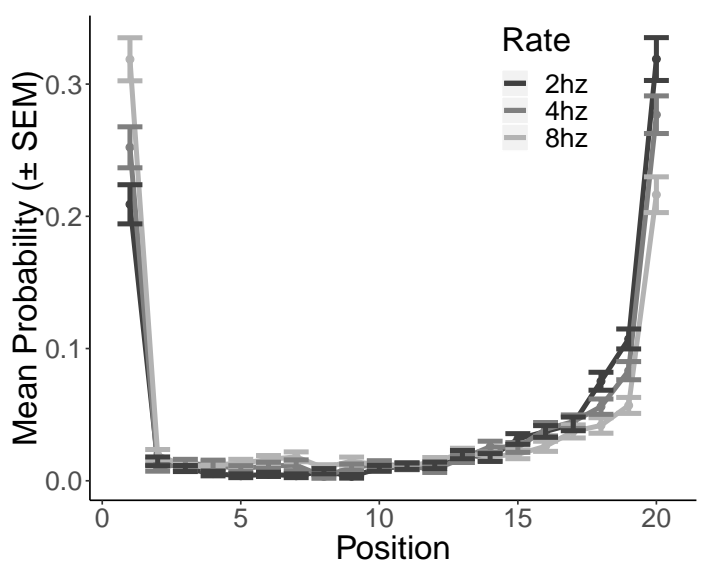

Figure 2: Probability of first recall. Participants tended to begin recall by naming an item from the beginning or end of the list. As presentation rates increased, the probability of initiating recall at the end of the list decreased, and the probability of initiating recall with at the beginning of the list increased.

Post-hoc paired permutations confirmed these results, showing that the presentation rate of $2 \mathrm{~Hz}$ yielded significantly higher number of recalls than $4 \mathrm{~Hz}(p<0.001$, Cohen's D $=0.9)$ and $8 \mathrm{~Hz}(p<0.001$, Cohen's $\mathrm{D}=1.5)$, and that $4 \mathrm{~Hz}$ produced significantly more recalls than $8 \mathrm{~Hz}(p<0.001$, Cohen's $\mathrm{D}=0.69$ ). This result is consistent with previous findings that faster presentation rates decrease the number of words recalled in a free recall task (B. B. Murdock Jr, 1960).

\section{Increasing Presentation Rates Increases the Primacy Effect and Decreases the Recency Effect}

Participants were more likely to begin recall by reporting a word at the beginning or end of the list (Figure 2). As the presentation rate increased, participants initiated recall less frequently at the end of the list and more frequently at the beginning of the list. This was confirmed by paired permutation tests which indicated that the probability of beginning a recall with the first item in a studied list was greater at 8 $\mathrm{Hz}$ than both $4 \mathrm{~Hz}(p<0.001$, Cohen's $D=0.24)$ and $2 \mathrm{~Hz}$ ( $p<0.001$, Cohen's $D=0.43$ ), and greater for $4 \mathrm{~Hz}$ than 2 $\mathrm{Hz}(p<0.001$, Cohen's $D=0.18)$. Conversely, the probability of first recalling the last item in a list was greater for 2 $\mathrm{Hz}$ than both $4 \mathrm{~Hz}(p=0.002$, Cohen's $D=0.16)$ and $8 \mathrm{~Hz}$ ( $p<0.001$, Cohen's $D=0.40)$, and higher for $4 \mathrm{~Hz}$ than $8 \mathrm{~Hz}$ $(p<0.001$, Cohen's $D=0.25)$.

As shown in Figure 3, participants showed a higher rate of recalling words from the beginning and end of a list compared to words in the middle (Figure 3). Consistent with previous findings, increasing presentation rates resulted in lower recall for the final item in the list. This was as confirmed by a paired permutation test which found that the probability of recalling the last item in a list was greater for $2 \mathrm{~Hz}$ than both $4 \mathrm{~Hz}(p<$ 0.001 , Cohen's $D=0.50)$ and $8 \mathrm{~Hz}(p<0.001$, Cohen's $D=$ $0.65)$, and greater for $4 \mathrm{~Hz}$ than $8 \mathrm{~Hz}(p=0.01$, Cohen's $D=$

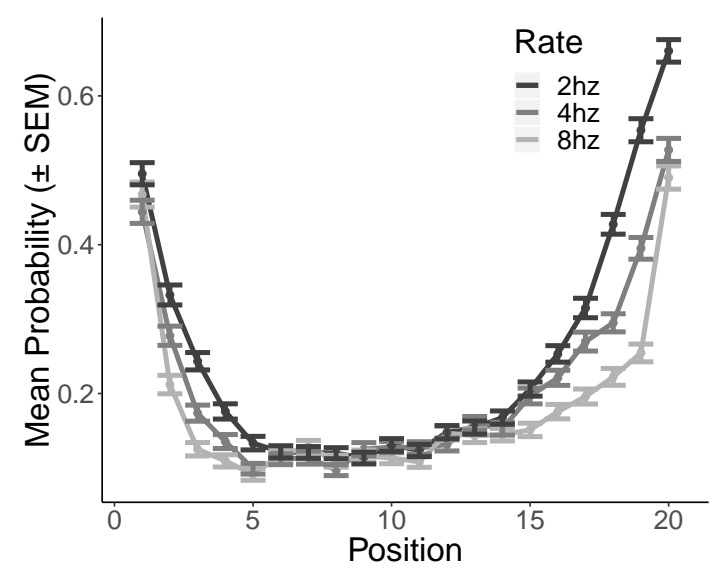

Figure 3: Probability of recall as a function of position in study list. Participants showed the highest level of performance for items at the beginning and end of the list. As presentation rate increased, participants showed a tendency to have a lower recency effect in comparison to the primacy effect.

0.15). In contrast to the PFR, increasing presentation rates did not improve the overall probability of recalling the first item in a list. Rather, it was found that the probability of recalling the first item of a list was greater at $2 \mathrm{~Hz}$ than $4 \mathrm{~Hz}$ ( $p=0.008$, Cohen's $D=0.17$ ), and otherwise there were no significant difference (all $p>0.05$ ).

\section{The Contiguity Effect Flattens At Higher Presentation Rates}

Figure 4 shows the number of transitions between each serial position in each of the three conditions. The primacy and recency effects can be readily distinguished, as is the tendency to make remote transitions to the beginning of the list. The contiguity effect can be seen as a slightly darker shade along the diagonal; the forward asymmetry appears as a darker shade just above the diagonal. As expected, the contiguity effect appeared to decrease as presentation rate increased. Because primacy and recency effects are a confound in identifying the contiguity effect we calculated the lag-CRP using only transitions that came from items from the middle of the list (serial positions 7-13).

Figure 5 displays the average probability of transitioning from a recalled word to a word at a given lag (with lag 0 corresponding to the diagonal of the matrices in Figure 4), and appears to show a reduction in the temporal contiguity effect as the presentation rate increases. We performed a mixed effect logistic regression to estimate the probability of recall based on absolute lag for each presentation rate separately. This showed that distance from the previously-recalled item significantly decreased the probability of recall for $2 \mathrm{~Hz}$ $(z=-9.74, p<0.001)$ and $4 \mathrm{~Hz}(z=-4.93, p<0.001)$, but not for $8 \mathrm{~Hz}(z=-0.70, p=0.48)$. We then computed another mixed effects logistic regression to test the interaction between absolute lag, its direction (backwards or for- 

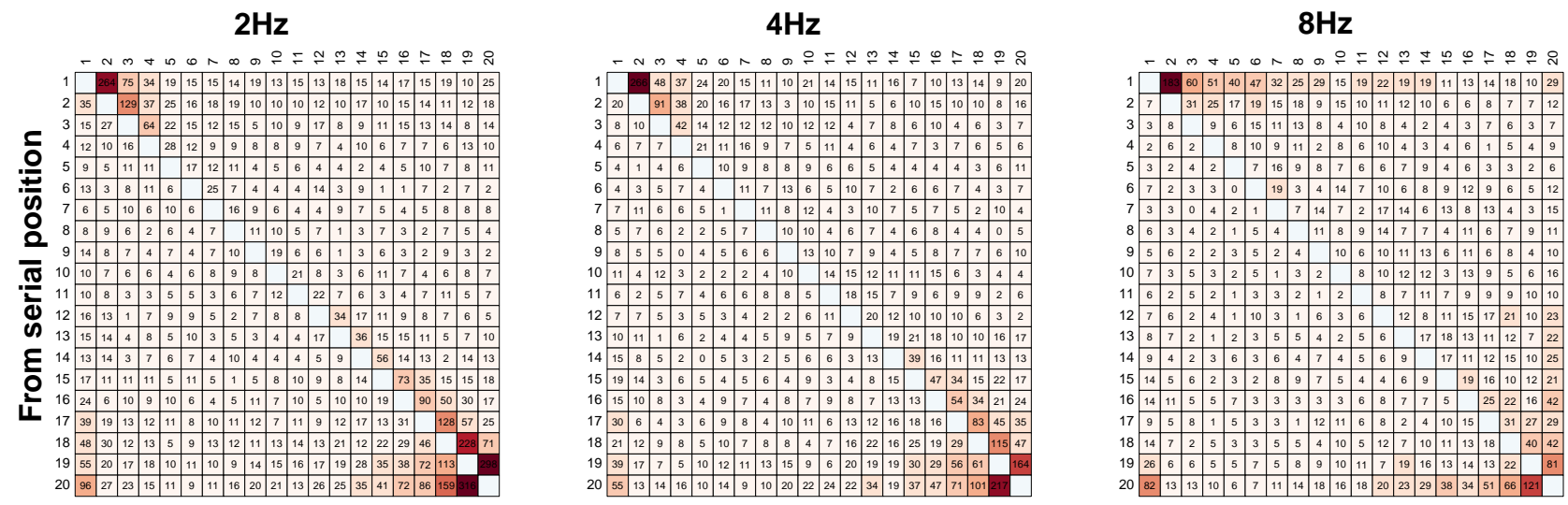

To serial position

Figure 4: Matrices showing the number of total valid recall transitions between any two study list positions for each presentation rate separately. Colors and numbers correspond to the number of such recalls summed across participants. Transitions between extreme positions in study lists correspond to primacy and recency effects, which persist across rates. In contrast, the likelihood of recalling nearby items (i.e., close to the diagonal) appears to decrease as presentation rate increases.

wards from the previously recalled item), and the presentation rate. This analysis showed that transitions in the forward direction were more probable than backward transitions $(z=4.18, p<0.001)$; transitions at more distant lags were less probable $(z=-4.83, p<0.001)$; probabilities were higher for $2 \mathrm{~Hz}$ compared to $4 \mathrm{~Hz}(z=-2.28, p=0.02)$ and $8 \mathrm{~Hz}(z=-3.63, p<0.001)$; the effect of absolute lag was stronger for forward transitions than backwards transitions $(z=-2.71, p<0.01)$, and the effect of lag was stronger for $2 \mathrm{~Hz}$ compared to $4 \mathrm{~Hz}(z=2.11, p=0.03)$ and $8 \mathrm{~Hz}$ $(z=3.12, p<0.01)$. All other interactions showed no significant effects (all $p>0.05$ ). These results show that increasing the presentation rate of studied words decreases the contiguity effect.

\section{Discussion}

Remembering past events is associated with a jump back in time, manifesting in a higher probability for temporally contiguous elements to be subsequently recalled. In this study, we investigated whether higher presentation rates would negatively impact the temporal contiguity effect. Many of our results were consistent with previous free recall studies. For instance, the average number of words recalled per list decreased as the presentation rate increased. Also, as the presentation rate increased, the recency effect was diminished. While the primacy effect increased in looking at the probability of first recall, there was not a clear effect on the overall probability of recalling the first item. The novel contribution of this paper is the finding that the temporal contiguity effect was disrupted by fast presentation rates, most notably in the $8 \mathrm{~Hz}$ condition. These findings suggest that encoding processes taking place on the order of 125 to $250 \mathrm{~ms}$ are im-

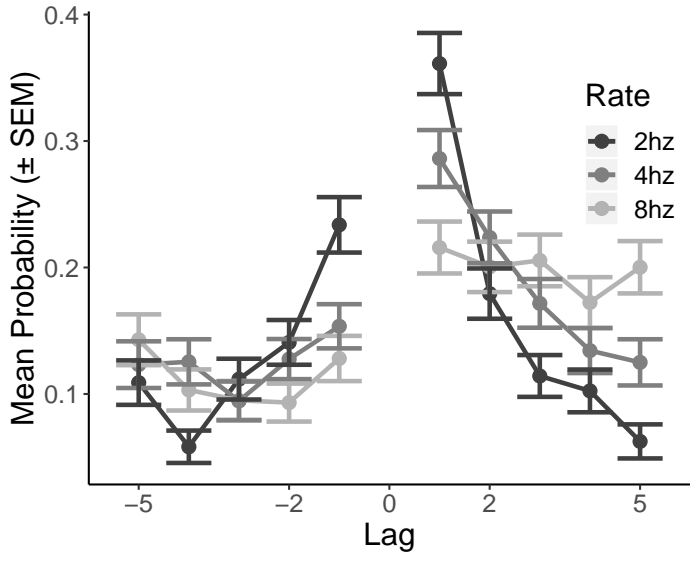

Figure 5: Lag-CRP for transitions restricted to serial positions 7-13 to avoid confounds with primacy and recency effects. As presentation rates increase, the contiguity effect weakens but remains asymmetric. At $8 \mathrm{~Hz}$ there is no positive evidence for a contiguity effect. 
portant for binding items to their temporal context.

Our results pose questions about the relation of presentation rate and neural coding. Medial temporal lobe theta (3$8 \mathrm{~Hz}$ ) is related to successful encoding in free recall, particularly when binding elements temporally (Nyhus \& Curran, 2010; Sederberg, Kahana, Howard, Donner, \& Madsen, 2003). In addition, Guderian, Schott, Richardson-Klavehn, and Düzel (2009) have shown that prediction of successfullyrecalled items relies on theta frequency. While presentation rates of $2 \mathrm{~Hz}$ and $4 \mathrm{~Hz}$ are mostly contained within this frequency band, $8 \mathrm{~Hz}$ lies at the upper bound of human theta. It is possible that presening eight words per second outpaces encoding processes that depend on theta (Hasselmo, Bodelón, \& Wyble, 2002), thus explaining why lag-CRPs become weaker for this presentation speed. Examination of encoding and retrieval periods using EEG and ECoG could help address this issue in the future.

\section{References}

Brady, T. F., Konkle, T., Alvarez, G. A., \& Oliva, A. (2008). Visual long-term memory has a massive storage capacity for object details. Proceedings of the National Academy of Sciences, 105(38), 14325-14329.

Davelaar, E. J., Goshen-Gottstein, Y., Ashkenazi, A., Haarmann, H. J., \& Usher, M. (2005). The demise of short-term memory revisited: empirical and computational investigations of recency effects. Psychological Review, 112(1), 342.

Donchin, E. (1981). Surprise!? surprise? Psychophysiology, 18(5), 493-513.

Friendly, M., Franklin, P. E., Hoffman, D., \& Rubin, D. C. (1982). The toronto word pool: Norms for imagery, concreteness, orthographic variables, and grammatical usage for 1,080 words. Behavior Research Methods \& Instrumentation, 14(4), 375-399.

Glanzer, M., \& Cunitz, A. R. (1966). Two storage mechanisms in free recall. Journal of Verbal Learning and Verbal Behavior, 5, 351-360.

Guderian, S., Schott, B. H., Richardson-Klavehn, A., \& Düzel, E. (2009). Medial temporal theta state before an event predicts episodic encoding success in humans. Proceedings of the National Academy of Sciences, 106(13), 5365-5370.

Hasselmo, M. E., Bodelón, C., \& Wyble, B. P. (2002). A proposed function for hippocampal theta rhythm: Separate phases of encoding and retrieval enhance reversal of prior learning. Neural Computation, 14, 793-817.

Healey, M. K., \& Kahana, M. J. (2014). Is memory search governed by universal principles or idiosyncratic strategies? Journal of Experimental Psychology: General, 143(2), 575.

Hogan, R. M. (1975). Interitem encoding and directed search in free recall. Memory \& Cognition, 3(2), 197-209.

Howard, M. W., \& Kahana, M. J. (1999). Contextual variability and serial position effects in free recall. Journal of
Experimental Psychology: Learning, Memory, and Cognition, 25, 923-941.

Howard, M. W., Youker, T. E., \& Venkatadass, V. (2008). The persistence of memory: Contiguity effects across several minutes. Psychonomic Bulletin \& Review, 15(PMC2493616), 58-63.

Kahana, M. J. (1996). Associative retrieval processes in free recall. Memory \& Cognition, 24, 103-109.

Kahana, M. J. (2012). Foundations of human memory. OUP USA.

Kahana, M. J., Howard, M. W., Zaromb, F., \& Wingfield, A. (2002). Age dissociates recency and lag-recency effects in free recall. Journal of Experimental Psychology: Learning, Memory, and Cognition, 28, 530-540.

Laming, D. (1999). Testing the idea of distinct storage mechanisms in memory. International Journal of Psychology, 34(5/6), 419-426.

Murdock, B. B. (1962). The serial position effect of free recall. Journal of Experimental Psychology, 64, 482-488.

Murdock, B. B., Jr. (1960). The immediate retention of unrelated words. Journal of Experimental Psychology, 60, 222-34.

Nyhus, E., \& Curran, T. (2010). Functional role of gamma and theta oscillations in episodic memory. Neuroscience and Biobehavioral Reviews, 34(7), 1023-35. doi: 10.1016/j.neubiorev.2009.12.014

Palombo, D. J., Di Lascio, J. M., Howard, M. W., \& Verfaellie, M. (2019). Medial temporal lobe amnesia is associated with a deficit in recovering temporal context. Journal of cognitive neuroscience, 31(2), 236-248.

Potter, M. C. (1976). Short-term conceptual memory for pictures. Journal of experimental psychology: human learning and memory, 2(5), 509.

Potter, M. C., \& Levy, E. I. (1969). Recognition memory for a rapid sequence of pictures. Journal of experimental psychology, 81(1), 10.

Sederberg, P. B., Kahana, M. J., Howard, M. W., Donner, E. J., \& Madsen, J. R. (2003). Theta and gamma oscillations during encoding predict subsequent recall. Journal of Neuroscience, 23(34), 10809-14.

Standing, L. (1973). Learning 10000 pictures. The Quarterly journal of experimental psychology, 25(2), 207-222.

Tulving, E. (1983). Elements of episodic memory. New York: Oxford.

Unsworth, N. (2008). Exploring the retrieval dynamics of delayed and final free recall: Further evidence for temporalcontextual search. Journal of Memory and Language, 59, 223-236.

Wyble, B., Bowman, H., \& Nieuwenstein, M. (2009). The attentional blink provides episodic distinctiveness: sparing at a cost. Journal of Experimental Psychology: Human Perception and Performance, 35(3), 787-807. 